\title{
The Quantum Well of One-Dimensional Photonic Crystals
}

\author{
Xiao-Jing Liu, ${ }^{1,2}$ Ji Ma, ${ }^{1}$ Xiang-Dong Meng, ${ }^{1}$ Hai-Bo Li, ${ }^{1}$ Jing-Bin Lu, ${ }^{2}$ Hong Li, ${ }^{1}$ \\ Wan-Jin Chen, ${ }^{1}$ Xiang-Yao Wu, ${ }^{1}$ Si-Qi Zhang, ${ }^{2}$ and Yi-Heng $\mathrm{Wu}^{3}$ \\ ${ }^{1}$ Institute of Physics, Jilin Normal University, Siping 136000, China \\ ${ }^{2}$ Institute of Physics, Jilin University, Changchun 130012, China \\ ${ }^{3}$ School of Physics and Electronic Engineering, Anqing Normal University, Anqing 246133, China \\ Correspondence should be addressed to Xiang-Dong Meng; bestben@163.com
}

Received 6 January 2015; Accepted 8 April 2015

Academic Editor: Ashok Chatterjee

Copyright (C) 2015 Xiao-Jing Liu et al. This is an open access article distributed under the Creative Commons Attribution License, which permits unrestricted use, distribution, and reproduction in any medium, provided the original work is properly cited.

\begin{abstract}
We have studied the transmissivity of one-dimensional photonic crystals quantum well (QW) with quantum theory approach. By calculation, we find that there are photon bound states in the QW structure $(B A)^{6}(B B A B B)^{n}(A B)^{6}$, and the numbers of the bound states are equal to $n+1$. We have found that there are some new features in the QW, which can be used to design optic amplifier, attenuator, and optic filter of multiple channel.
\end{abstract}

\section{Introduction}

Photonic crystals (PCs) are artificial structures with a periodic dielectric constant in one, two, or three dimensions $[1,2]$. They are characterized by photonic band structures owing to the multiple Bragg scatterings [3, 4]. Between photonic bands there may exist a photonic band gap (PBG), in which the propagation of electromagnetic waves or photons is strongly inhibited [5]. This facilitates the manipulation and control of the flow of electromagnetic waves or photons as well as the design of high-performance optoelectric devices $[6,7]$.

The concept of super lattice and quantum well (QW) stemmed from the pioneering work of Shimuzu and Ishihara [8]. It is well known that there are many interesting and new phenomena for electrons in semiconductor QW structures [9]. The QW structures and super lattices can be used to tailor the electronic band structures of semiconductors [913]. Similar to the idea of semiconductor QW structures, one can use different PCs to construct photonic QW structures, provided that the PBG of the constituent PCs are aligned properly. The constituents can be one-dimensional (1D), two-dimensional (2D), or three-dimensional (3D) PCs. It has been shown by the authors [14] that the transmission properties of the $1 \mathrm{D}$ and 2D PCs can be tailored by using QW structures. The nontransmission frequency range can be enlarged as desired by using QW. The use of QW exciton embedded in high-finesse semiconductor microcavities of the Fabry-Perot type has allowed observing a modification of spontaneous emission (weak coupling regime) [15-21] as well as the occurrence of a vacuum Rabi splitting (strong coupling regime) [22-25]. The latter effect arises when the radiationmatter coupling energy overcomes the damping rates of QW exciton and microcavities photons.

In $[26,27]$, we have studied the quantum transmission characteristic of $1 \mathrm{D}$ PCs with quantum theory approach and given the quantum transform matrix, quantum transmissivity, and reflectivity. In this paper, we use the quantum method to research the QW transmissivity of $1 D$ PCs. It is found that there are some new features in the QW structure $(B A)^{6}(B B A B B)^{n}(A B)^{6}$, which can be used to design optic amplifier, attenuator, and optic filter of multiple channel.

\section{Quantum Transform Matrix and Transmissivity of QW}

The QW structures consisted of two different 1D PCs. The first and second $1 D$ PCs structures are $(B A)^{6}(A B)^{6}$ and $(B B A B B)^{n}$, respectively, where $n$ are the numbers of the second PCs layers. The two $1 D$ PCs can consist of $1 D$ PCs of QW structure, which is $(B A)^{6}(B B A B B)^{n}(A B)^{6}$. 


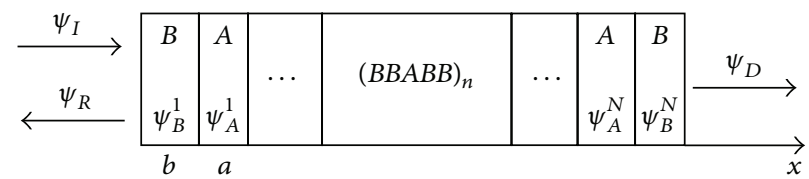

Figure 1: The QW structure of 1D PCs.

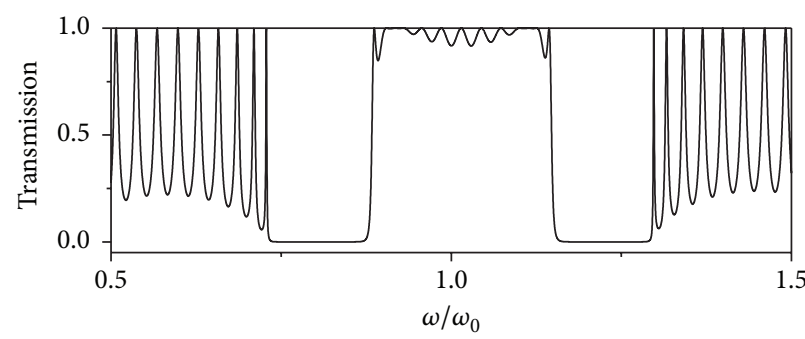

(a)

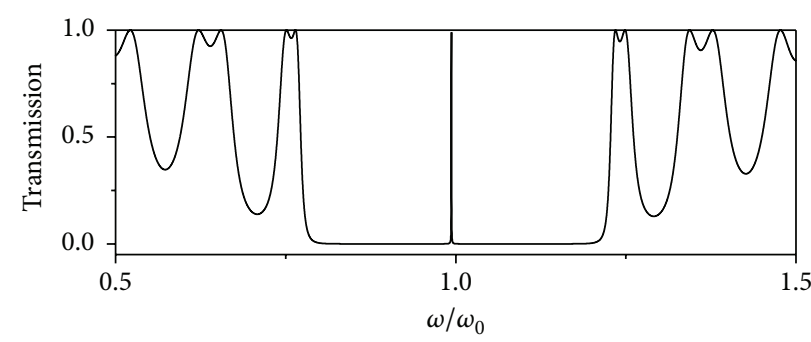

(b)

Figure 2: The quantum transmissivity of $1 \mathrm{D}$ PCs with $n_{b}=2.45$. (a) The structure is $(B B A B B)^{12}$; (b) the structure is $(B A)^{6}(A B)^{6}$.

In quantum theory approach $[26,27]$, we consider the photon travels along with the $x$-axis, and the QW structure and quantum wave functions distribution are shown in Figure 1. The thicknesses and refractive indexes of layers $A$ and $B$ are $a, b, n_{a}$, and $n_{b}$, respectively. The $\psi_{B}^{1}$ and $\psi_{A}^{1}$ are the photon wave functions of the first period media $B$ and $A$. The photon wave functions of incident, reflection, and transmission are $[26,27]$

$$
\begin{aligned}
\psi_{I} & =F e^{i K x}, \\
\psi_{R} & =F^{\prime} e^{-i K x}, \\
\psi_{D} & =D e^{i k_{A} P a+i k_{B} Q b+K(x-P a-Q b),}
\end{aligned}
$$

where $K=\omega / c, k_{A}=(\omega / c) n_{a}$, and $k_{B}=(\omega / c) n_{b}$ are the wave vector of photon in vacuum, mediums $A$ and $B$. The constants $F, F^{\prime}$, and $D$ are the wave function amplitudes of incident, reflection, and transmission wave. By calculation, similarly as $[26,27]$, we can directly give the wave functions of photon in arbitrary layers $A$ and $B$. For medium $A(B)$ of the $\left(P_{i}+1\right)$ th $\left(\left(Q_{j}+1\right)\right.$ th) layer, the photon wave function can be written as

$$
\begin{aligned}
\psi_{A}^{P_{i}+1}(x)= & A e^{i\left[k_{A} P_{i} a+k_{B} Q_{i} b+k_{A}\left(x-P_{i} a-Q_{i} b\right)\right]} \\
& +A^{\prime} e^{i\left[k_{A}\left(P_{i}+1\right) a+k_{B} Q_{i} b+k_{A}\left(\left(P_{i}+1\right) a+Q_{i} b-x\right)\right]}, \\
\psi_{B}^{Q_{j}+1}(x)= & B e^{i\left[k_{A} P_{j} a+k_{B} Q_{j} b+k_{B}\left(x-P_{j} a-Q_{j} b\right)\right]} \\
& +B^{\prime} e^{i\left[k_{A} P_{j} a+k_{B}\left(Q_{j}+1\right) b+k_{B}\left(P_{j} a+\left(Q_{j}+1\right) b-x\right)\right]} ;
\end{aligned}
$$

before the $\left(P_{i}+1\right)$ th layer medium $A$, there are $P_{i}$ layers medium $A$ and $Q_{i}$ layers medium $B$, and before the $\left(Q_{j}+1\right)$ th layer medium $B$, there are $P_{j}$ layers medium $A$ and $Q_{j}$ layers medium $B$. The constants $A, A^{\prime}, B$, and $B^{\prime}$ are the wave function amplitudes. By the condition of wave function and its derivative continuation at the interface of two mediums, we can obtain the quantum transfer matrix of $j$ th medium layer; it is

$$
M_{j}=\frac{1}{2}\left(\begin{array}{cc}
1+\frac{k_{j-1}}{k_{j}} & 1-\frac{k_{j-1}}{k_{j}} \\
\left(1-\frac{k_{j-1}}{k_{j}}\right) e^{-2 i k_{j} d_{j}} & \left(1+\frac{k_{j-1}}{k_{j}}\right) e^{-2 i k_{j} d_{j}}
\end{array}\right),
$$

where $k_{j}\left(k_{j-1}\right)$ is the wave vector of photon in the $j$ th $\left((j-1)\right.$ th) layer medium and $d_{j}$ is the thickness of $j$ th layer medium. For the QW structure $(B A)^{6}(B B A B B)^{n}(A B)^{6}$, its total quantum transfer matrix is

$$
\begin{aligned}
M & =\left(M_{B} M_{A}\right)^{6}\left(M_{B} M_{B} M_{A} M_{B} M_{B}\right)^{n}\left(M_{A} M_{B}\right)^{6} \\
& =\left(\begin{array}{ll}
m_{1} & m_{2} \\
m_{3} & m_{4}
\end{array}\right),
\end{aligned}
$$

and the quantum transmissivity $T$ is $[26,27]$

$$
T=\left|\frac{D}{F}\right|^{2}=\left|\frac{2 k_{A}\left(m_{2} m_{3}-m_{1} m_{4}\right)}{m_{2}\left(k_{A}-K\right)-m_{4}\left(k_{A}+K\right)}\right|^{2} .
$$

\section{Numerical Result}

In this section, we report our numerical results of the QW quantum transmissivity. The refractive indexes and the thicknesses of medium $A$ and medium $B$ are as follows: $n_{a}=1.35, n_{b}=2.45$ and $a=890 \mathrm{~nm}, b=469 \mathrm{~nm}$. The quantum transmissivity of $(B B A B B)^{12}$ and $(B A)^{6}(A B)^{6}$ is shown in Figures 2(a) and 2(b). In Figures 3 and 4, we only change the refractive index of medium $B$ in relation to Figure 2; they are $n_{b}=2.45-i 0.0001$ (active medium) and $n_{b}=2.45+i 0.0001$ (absorbing medium). From Figures 2 to 4, we can obtain results as follows. (1) In Figures 2(b), $3(\mathrm{~b})$, and $4(\mathrm{~b})$, when the ratio $\omega / \omega_{0}=1.0$, the quantum transmission peaks are $T=1.0, T>1.5$, and $T<1.0$ for 


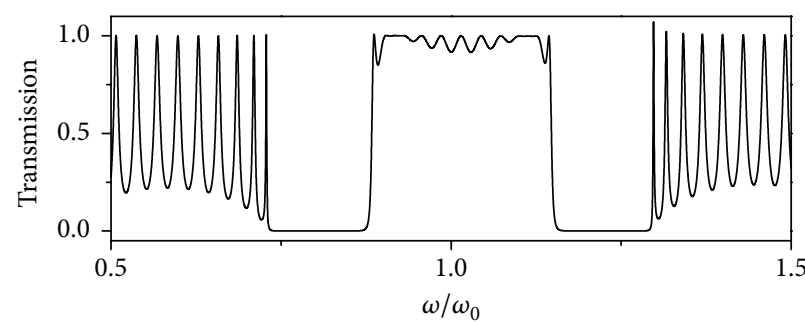

(a)

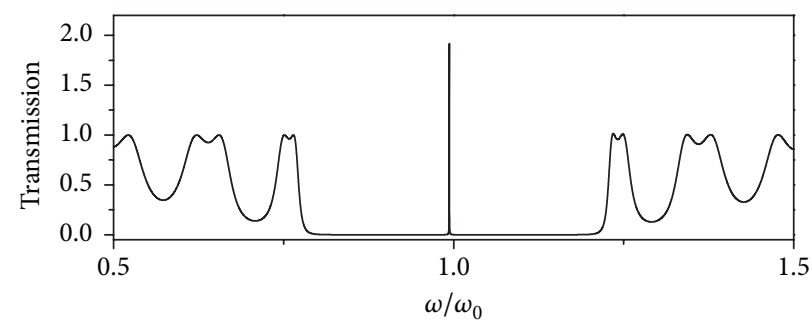

(b)

Figure 3: The quantum transmissivity of $1 \mathrm{D}$ PCs with $n_{b}=2.45-i 0.0001$. (a) The structure is $(B B A B B)^{12}$; (b) the structure is $(B A)^{6}(A B)^{6}$.

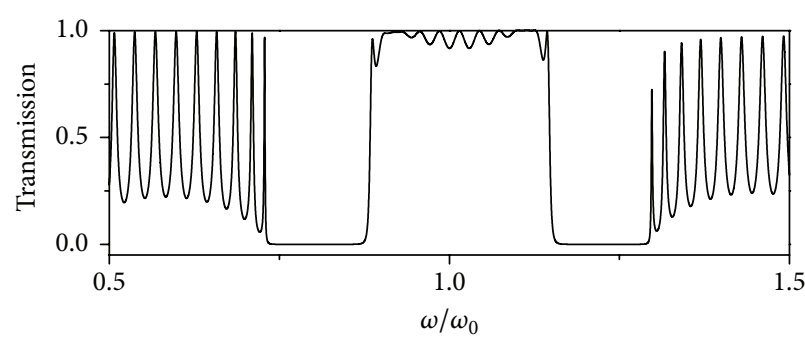

(a)

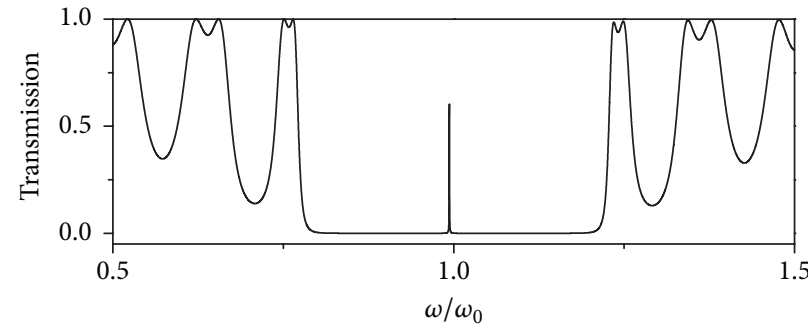

(b)

FIgURE 4: The quantum transmissivity of $1 \mathrm{D}$ PCs with $n_{b}=2.45+i 0.0001$. (a) The structure is $(B B A B B)^{12} ;(\mathrm{b})$ the structure is $(B A)^{6}(A B)^{6}$.

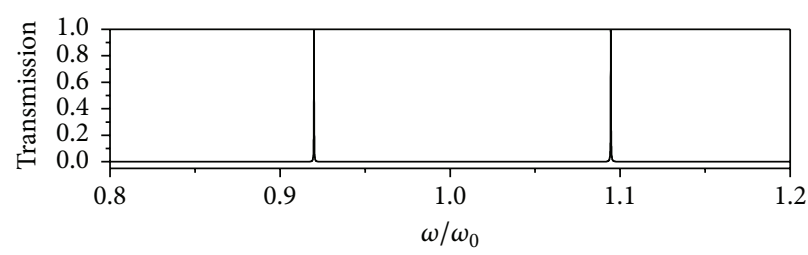

(a)

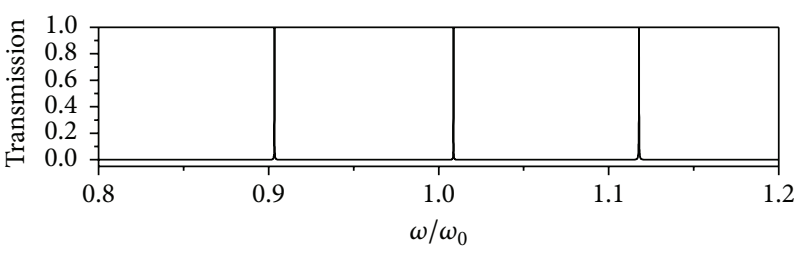

(b)

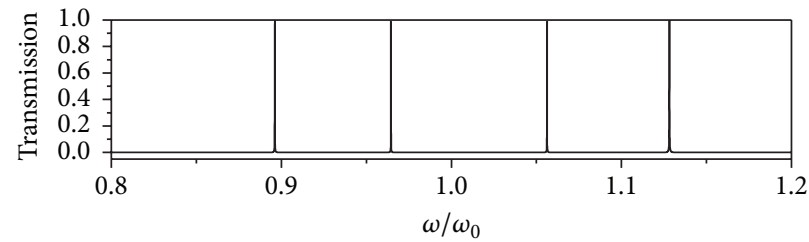

(c)

Figure 5: The quantum transmissivity for QW structure $(B A)^{6}(B B A B B)^{n}(A B)^{6}$ with $n_{b}=2.45$. (a) $n=1$, (b) $n=2$, and (c) $n=3$.

the $1 \mathrm{D}$ PCs $(B A)^{6}(A B)^{6}$; that is, the quantum transmission peaks are gained and attenuated when the medium $B$ is active medium $\left(n_{b}=2.45-i 0.0001\right)$ and absorbing medium $\left(n_{b}=2.45+i 0.0001\right)$. (2) The forbidden band of 1D PCs $(B A)^{6}(A B)^{6}$ is in the range of $\omega / \omega_{0}=0.8 \sim 1.2$, and the conduction band of $1 \mathrm{D}$ PCs $(B B A B B)^{12}$ is inside the forbidden band. So, the PCs $(B A)^{6}(A B)^{6}$ play a role similar to a barrier to PCs $(B B A B B)^{12}$, and the PCs $(B B A B B)^{12}$ act as a well in the forbidden band. We put the 1D PCs $(B B A B B)^{n}$ and $(B A)^{6}(A B)^{6}$ together to constitute the $1 \mathrm{D}$ PCs of QW structure $(B A)^{6}(B B A B B)^{n}(A B)^{6}$, which are shown in Figure 1. In Figures 5, 6, and 7, we should study the quantum transmissivity of the QW structure $(B A)^{6}(B B A B B)^{n}(A B)^{6}$. As mentioned above, the conduction band of PCs $(B B A B B)^{n}$ is inside the forbidden band of PCs $(B A)^{6}(A B)^{6}$; that is, the PCs $(B A)^{6}(A B)^{6}$ prohibit the propagation of photon in its forbidden band; then the photon will be confined in the PCs $(B B A B B)^{n}$. Because of the quantum effect of photon in QW of 1D PCs, the photon should form the bound state in the QW, which is analogous to the bound state of electron in semiconductor QW. The photon can pass the QW by the resonance perforation way and form the very sharp peaks of quantum transmissivity within the forbidden band of the PCs $(A B)^{6}(B A)^{6}$, which are shown in Figures 5, 6, and 7. In 


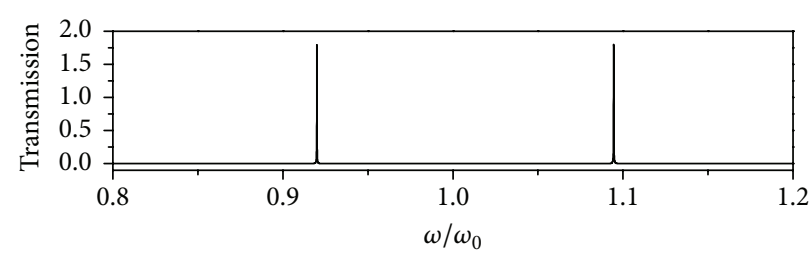

(a)

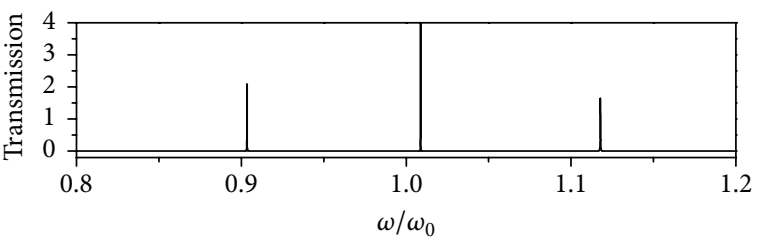

(b)

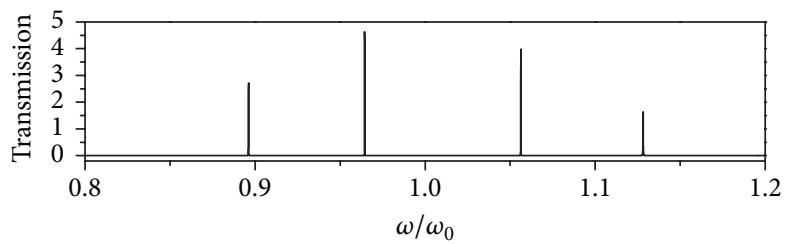

(c)

FIGURE 6: The quantum transmissivity for QW structure $(B A)^{6}(B B A B B)^{n}(A B)^{6}$ with $n_{b}=2.45-i 0.0001$. (a) $n=1$, (b) $n=2$, and (c) $n=3$.

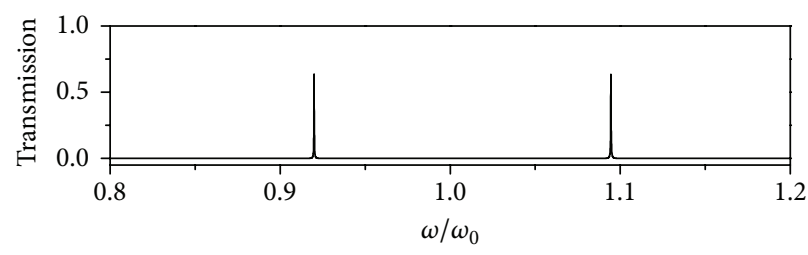

(a)

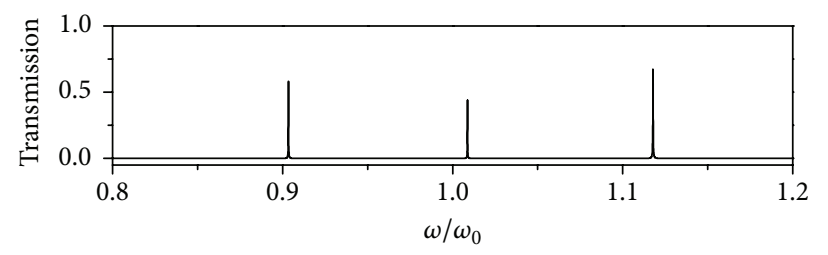

(b)

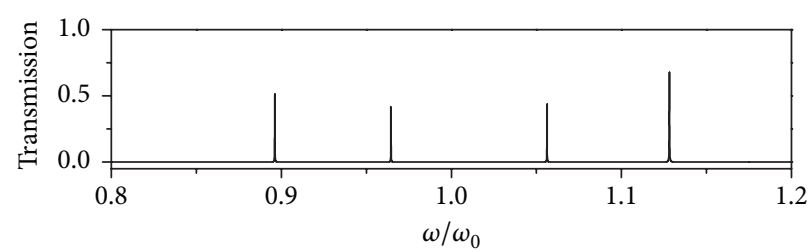

(c)

Figure 7: The quantum transmissivity for QW structure $(B A)^{6}(B B A B B)^{n}(A B)^{6}$ with $n_{b}=2.45+i 0.0001$. (a) $n=1$, (b) $n=2$, and (c) $n=3$.

Figures 5, 6, and 7, (a), (b), and (c) are corresponding to $n=1$, $n=2$, and $n=3$ for the QW structure $(B A)^{6}(B B A B B)^{n}(A B)^{6}$. In Figures 5, 6, and 7 refractive indexes of medium $B$ are real number, $n_{b}=2.45$ (convention medium), and complex numbers $n_{b}=2.45-i 0.0001$ (active medium) and $n_{b}=2.45+$ i0.0001 (absorbing medium), respectively. From Figures 5 to 7 , we can obtain some results. (1) The numbers of the sharp peaks (bound states) are equal to $n+1$; that is, when $n=1$, $n=2$, and $n=3$, the numbers of the sharp peaks are 2 , 3 , and 4. (2) In Figure 5, the quantum transmissivity of the sharp peaks $T=1$ for $n=1, n=2$, and $n=3$, which can be designed optic filter of multiple channel. (3) In Figure 6, the quantum transmissivity of the sharp peaks $T>1$ for $n=1$, $n=2$, and $n=3$. When $n$ increase, the sharp peaks value $T$ increase, which can be used to design optic amplifier and optic filter of multiple channel. (4) In Figure 7, the quantum transmissivity of the sharp peaks $T<1$ for $n=1, n=2$, and $n=3$; when $n$ increase, the sharp peaks value $T$ decrease, which can be used to design optic attenuator.

\section{Conclusion}

In summary, we have studied the quantum transmissivity of the QW of 1D PCs with quantum theory approach. By calculation, we find that there are photon bound states in QW structure $(B A)^{6}(B B A B B)^{n}(A B)^{6}$, and the numbers of the bound states are equal to $n+1$, which are formed by the quantum effect of photon in QW. We also find that the QW $(B A)^{6}(B B A B B)^{n}(A B)^{6}$ can be used to design optic amplifier, attenuator, and optic filter of multiple channel.

\section{Conflict of Interests}

The authors declare that there is no conflict of interests regarding the publication of this paper.

\section{Acknowledgments}

This work was supported by the National Natural Science Foundation of China (no. 61275047), the Research Project of 
Chinese Ministry of Education (no. 213009A), and Scientific and Technological Development Foundation of Jilin Province (no. 20130101031JC).

\section{References}

[1] C. M. Bowden, J. P. Dowling, and H. O. Everitt, "Development and applications of materials exhibiting photonic band gaps," Journal of the Optical Society of America B, vol. 10, pp. 280-282, 1993.

[2] A. Reinhard, T. Volz, M. Winger et al., "Strongly correlated photons on a chip," Nature Photonics, vol. 6, no. 2, pp. 93-96, 2012.

[3] J.-T. Liu, T.-B. Wang, X.-J. Li, and N.-H. Liu, "Enhanced absorption of monolayer $\mathrm{MoS}_{2}$ with resonant back reflector," Journal of Applied Physics, vol. 115, Article ID 193511, 2014.

[4] C. Weisbuch, M. Nishioka, A. Ishikawa, and Y. Arakawa, "Observation of the coupled exciton-photon mode splitting in a semiconductor quantum microcavity," Physical Review Letters, vol. 69, article 3314, 1992.

[5] D. Ballarini, M. de Giorgi, E. Cancellieri et al., "All-optical polariton transistor," Nature Communications, vol. 4, article 1778, 2013.

[6] G. Kurizki and J. W. Haus, "Special issue on photonic band structures," Journal of Modern Optics, vol. 41, no. 2, pp. 171-172, 1994.

[7] N. M. R. Peres and Y. V. Bludov, "Enhancing the absorption of graphene in the terahertz range," EPL, vol. 101, no. 5, Article ID 58002, 2013.

[8] M. Shimuzu and T. Ishihara, "Subpicosecond transmission change in semiconductor-embedded photonic crystal slab: toward ultrafast optical switching," Applied Physics Letters, vol. 80, no. 16, pp. 2836-2838, 2002.

[9] H. W. Tan, H. M. van Driel, S. L. Schweizer, R. B. Wehrspohn, and U. Gösele, "Nonlinear optical tuning of a two-dimensional silicon photonic crystal," Physical Review B, vol. 70, no. 20, Article ID 205110, 2004.

[10] F. D. M. Haldane and S. Raghu, "Possible realization of directional optical waveguides in photonic crystals with broken time-reversal symmetry," Physical Review Letters, vol. 100, no. 1, Article ID 013904, 4 pages, 2008.

[11] S. Raghu and F. D. M. Haldane, "Analogs of quantum-Halleffect edge states in photonic crystals," Physical Review A, vol. 78, Article ID 033834, 2008.

[12] Z. Wang, Y. Chong, J. D. Joannopoulos, and M. Soljačić, “Observation of unidirectional backscattering-immune topological electromagnetic states," Nature, vol. 461, no. 7265, pp. 772-775, 2009.

[13] Y. Poo, R.-X. Wu, Z. Lin, Y. Yang, and C. T. Chan, "Experimental realization of self-guiding unidirectional electromagnetic edge states," Phys. Rev. Lett, vol. 106, no. 9, Article ID 093903, 4 pages, 2011.

[14] J. P. Prineas, J. Y. Zhou, J. Kuhl et al., "Ultrafast ac Stark effect switching of the active photonic band gap from Bragg-periodic semiconductor quantum wells," Applied Physics Letters, vol. 81, no. 23, pp. 4332-4334, 2002.

[15] G. Björk, S. MacHida, Y. Yamamoto, and K. Igeta, "Modification of spontaneous emission rate in planar dielectric microcavity structures," Physical Review A, vol. 44, no. 1, pp. 669-681, 1991.

[16] K. Fang, Z. Yu, and S. Fan, "Realizing effective magnetic field for photons by controlling the phase of dynamic modulation," Nature Photonics, vol. 6, no. 11, pp. 782-787, 2012.
[17] M. C. Rechtsman, J. M. Zeuner, Y. Plotnik et al., "Photonic Floquet topological insulators," Nature, vol. 496, no. 7444, pp. 196-200, 2013.

[18] J. Cho, D. G. Angelakis, and S. Bose, "Fractional quantum Hall state in coupled cavities," Physical Review Letters, vol. 101, Article ID 246809, 2008.

[19] J. Koch, A. A. Houck, K. L. Hur, and S. M. Girvin, "Timereversal-symmetry breaking in circuit-QED-based photon lattices," Physical Review A, vol. 82, Article ID 043811, 2010.

[20] R. O. Umucalilar and I. Carusotto, "Artificial gauge field for photons in coupled cavity arrays," Physical Review A, vol. 84, no. 4, Article ID 043804, 8 pages, 2011.

[21] M. Hafezi, E. A. Demler, M. D. Lukin, and J. M. Taylor, "Robust optical delay lines with topological protection," Nature Physics, vol. 7, no. 11, pp. 907-912, 2011.

[22] M. Minkov and V. Savona, "Long-distance radiative excitation transfer between quantum dots in disordered photonic crystal waveguides," Physical Review B, vol. 88, Article ID 081303, 2013.

[23] R. Houdré, C. Weisbuch, R. P. Stanley, U. Oesterle, P. Pellandini, and M. Ilegems, "Measurement of cavity-polariton dispersion curve from angle-resolved photoluminescence experiments," Physical Review Letters, vol. 73, no. 15, pp. 2043-2046, 1994.

[24] M. Hafezi, S. Mittal, J. Fan, A. Migdall, and J. M. Taylor, "Imaging topological edge states in silicon photonics," Nature Photonics, vol. 7, no. 12, pp. 1001-1005, 2013.

[25] G. Q. Liang and Y. D. Chong, "Optical resonator analog of a twodimensional topological insulator," Physical Review Letters, vol. 110, Article ID 203904, 2013.

[26] X.-Y. Wu, J. Ma, X.-J. Liu et al., "Quantum theory of photonic crystals," Physica E: Low-Dimensional Systems and Nanostructures, vol. 59, pp. 174-180, 2014.

[27] X.-Y. Wu, J. Ma, X.-J. Liu et al., "Study photonic crystals defect model property with quantum theory," Optics Communications, vol. 321, pp. 211-218, 2014. 

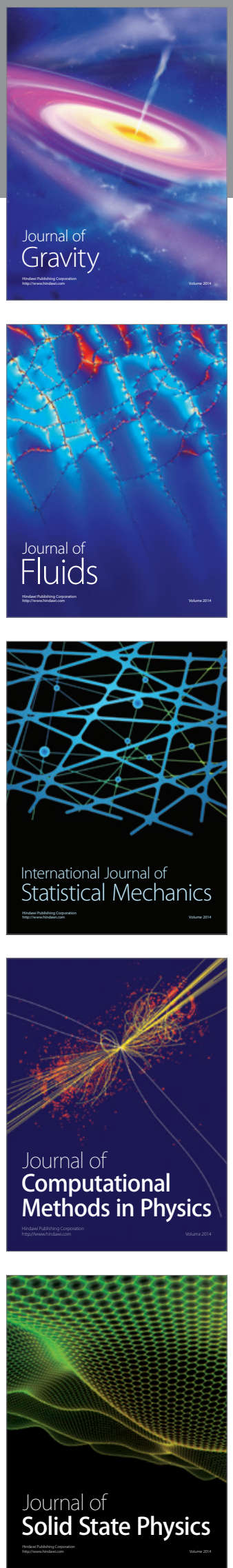

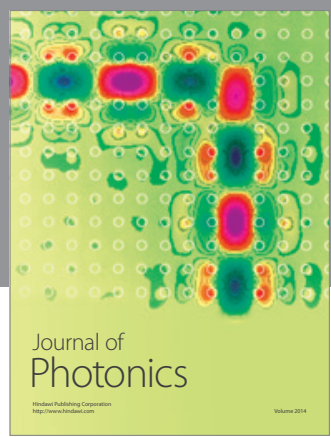

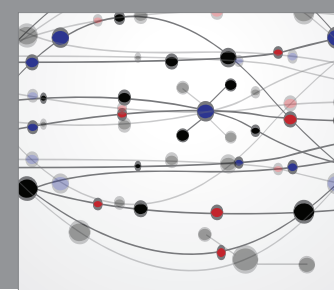

The Scientific World Journal

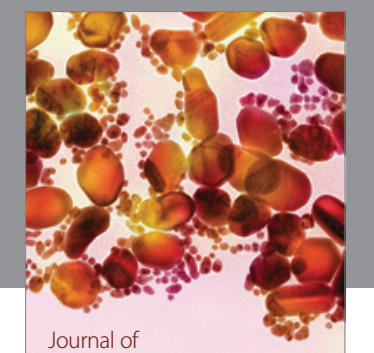

Soft Matter
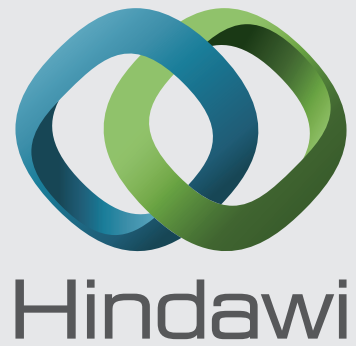

Submit your manuscripts at

http://www.hindawi.com
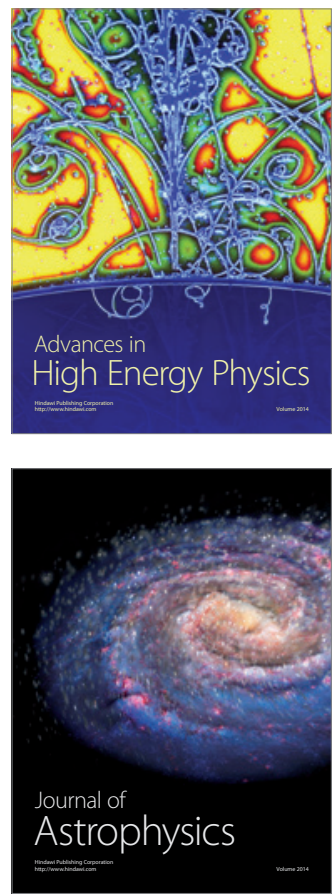
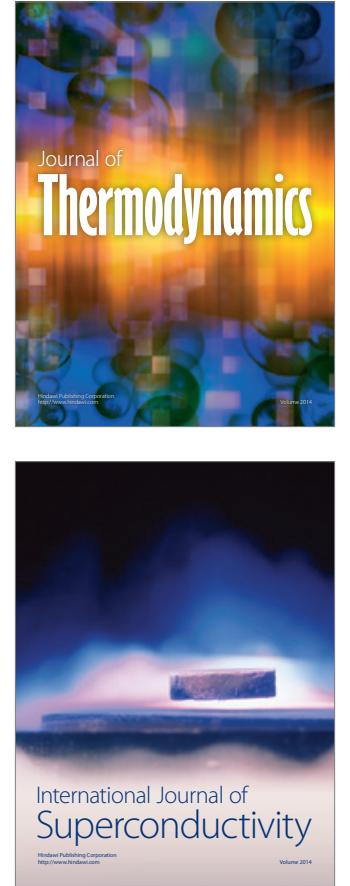
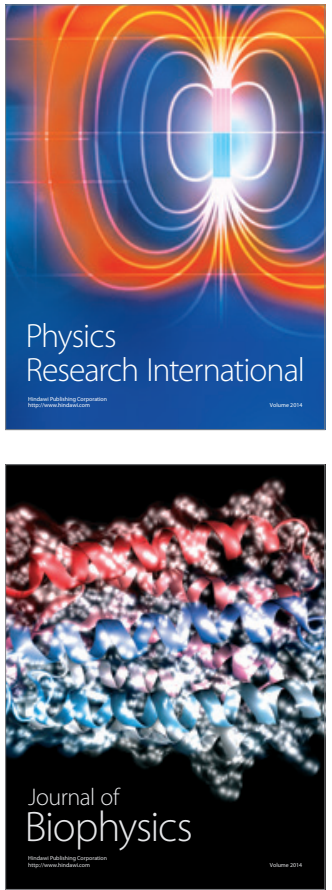
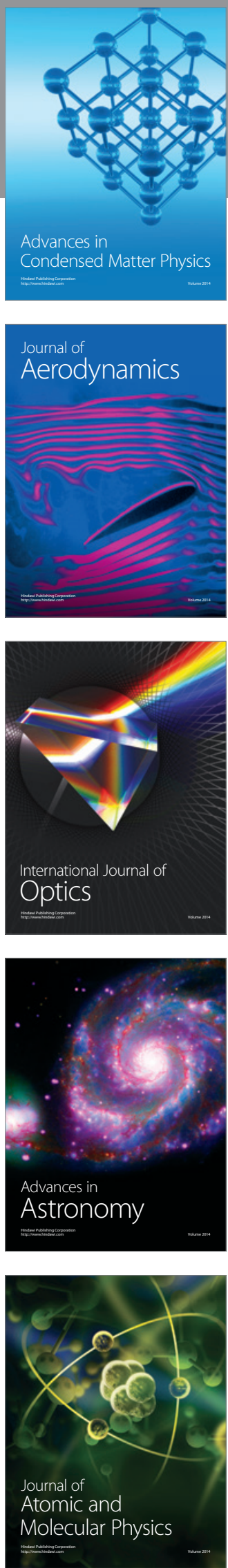